\title{
In situ fabrication of graphene from a copper-carbon nanoneedle and its electrical properties
}

\begin{abstract}
Herein, we present a direct observation of the formation of graphene from a single coppercarbon nanoneedle $(\mathrm{Cu}-\mathrm{CNN})$ during the measurement of current-voltage (I-V) and direct heating via in situ transmission electron microscopy (TEM). Significant structural transformation of $\mathrm{Cu}-\mathrm{CNN}$ was observed with an applied potential in a two probe system. Under a high current flow between $4.9 \mu \mathrm{A}$ to $49.0 \mu \mathrm{A}$, the $\mathrm{Cu}$ nanoparticles melted and evaporated due to Joule heating. The amorphous carbon began crystallizing and transformed into $\mathrm{sp}^{2}$ hybridized hollow graphitic carbon, which was catalyzed by the dispersed $\mathrm{Cu}$ nanoparticles. The temperature generated during the current flow was estimated to be 1073 $\mathrm{K}$, as revealed by an in situ TEM heating experiment. The graphene nanoneedle formed exhibited a high current density of $10^{6} \mathrm{~A} \mathrm{~cm}^{-2}$, which is comparable to $\mathrm{Cu}$ in normal interconnect applications. Thus, the graphene nanoneedle formed will be promising for future alternative interconnect materials.
\end{abstract}

Keyword: In situ fabrication; Graphene; Copper-carbon nanoneedle; Eectrical properties 